\title{
Submitted: 7 Role of intrapartum ultrasound in modern obstetrics - Accepted: current perspectives and literature review
} 11.11.2019

Published: 31.12.2019

\section{Keywords}

intrapartum ultrasound, transperineal ultrasound, angle of progression, labour

\author{
Małgorzata Siergiej ${ }^{1}$, Iwona Sudoł-Szopińska², Jerzy Zwoliński \\ Anna Maria Śladowska-Zwolińska ${ }^{1}$ \\ ${ }^{1}$ Department of Obstetrics, Holy Family Hospital, Warsaw, Poland \\ ${ }^{2}$ National Institute of Geriatrics, Rheumatology and Rehabilitation, Warsaw, Poland \\ Correspondence: Matgorzata Siergiej, Department of Obstetrics, Holy Family Hospital, \\ Madalińskiego 25, 02-544 Warsaw; tel.: +48 694004 181, \\ e-mail:malgorzata.siergiej@gmail.com
}

DOI: $10.15557 / \mathrm{JoU} .2019 .0044$

\begin{abstract}
For many years, the progress of labour has been traditionally evaluated almost exclusively by transvaginal digital assessment which, by its very nature, is an imprecise and, above all, subjective examination. Appropriate assessment of foetal head station and position in the birth canal is of critical importance for predicting further progress and safe completion of labour by instrumental or surgical intervention. In view of the deficiency of diagnostic methods available in the delivery room, attempts are undertaken to introduce intrapartum ultrasound performed using a transabdominal suprapubic or transperineal approach as a useful diagnostic tool. The examination is performed at the patient's bedside, using a portable ultrasound unit equipped with a convex probe. The method comprises a range of parameters, of which the most common are the angle of progression (AoP), foetal head direction, headperineum distance or midline angle (MLA). Intrapartum sonography yields an array of data to evaluate with a high degree of precision the foetal head position and station in the birth canal. Intrapartum ultrasound may prove a very useful method complementing traditional obstetric examination in a number of clinical situations such as prolonged delivery and lack of certainty as to the way to end the labour. Increasingly, attention is being drawn to the role of the examination in predicting the efficacy of induction of labour, serving as visual biofeedback to increase the effectiveness of maternal pushing or accurately identify the beginning of labour. It has been highlighted that intrapartum ultrasound is easy to use, painless, and reproducible. Also, the method does not require specialist training. Despite promising research results and the development of recommendations on the application of the method, there is still insufficient evidence to elaborate definite algorithms for the interpretation of results, based on which clinical decisions could be made.
\end{abstract}

\section{Introduction}

More than half a century has passed since Ian Donald began his pioneering research into the use of ultrasound in pregnant women ${ }^{(1,2)}$. Since then, ultrasonography has systematically grown in relevance in gynaecological and obstetric practice, acquiring the status of an effective diagnostic method. The role of ultrasound examinations in various gynaecological and obstetric applications has been consistently growing, which naturally entails an improved experience in the use of the technique, the interpretation of images, and the performance of a range of procedures (e.g. amniocentesis or cordocentesis) under ultrasound control. 
The only place where ultrasonography has not, as yet, found widespread applications is the delivery room.

\section{Traditional assessment of labour progress}

Labour management has changed little over the past decades, and it is still based on transvaginal digital examination which, by definition, is subjective and hence associated with a high rate of error. The learning curve for performing manual obstetrical examination according to conventional textbooks is long ${ }^{(3)}$. While the assessment of cervical dilation does not usually pose major problems, the exact determination of foetal head position and station in the birth canal may be problematic even for specialists with long obstetric experience.

In studies based on birth simulators, cervical dilation was determined precisely only in $56 \%$ of transvaginal digital assessments. What is more, the number of errors made by the same examiners exceeded $50 \%{ }^{(4)}$. In other studies, using a model made of soft materials to make it more realistic, cervical dilation was assessed correctly only in $19 \%$ of simulated examinations ${ }^{(5)}$. The accuracy of obstetric examination also depends on the experience of the clinician. One study found that the estimation of cervical dilatation performed by obstetricians with extensive experience were consistent with the findings obtained by a beginner physician in less than half of all examinations ${ }^{(6)}$.

As shown in the literature, the assessment of foetal head station in the birth canal is equally problematic to the examining physicians. In studies using a birth simulator, one in three obstetricians erroneously assessed foetal head position ${ }^{(7)}$. Sherer et al. compared the accuracy of foetal head position assessment by transvaginal digital examination and transabdominal ultrasound. Consistent findings were obtained in only $35 \%$ of patients in the second stage of labour ${ }^{(8)}$, and in $24 \%$ of parturient women in the active phase of labour defined as cervical dilation of at least $4 \mathrm{~cm}^{(9)}$. Based on the same method, other authors obtained the following results: $65 \%$ in the second stage, and $31 \%$ in the first stage of labour. It was also noted that the assessment of foetal head position in the birth canal was impossible in as many as $60 \%$ of patients in the first, and $30 \%$ of patients in the second stage of labour ${ }^{(10,11)}$.

Some researchers evaluated the accuracy of transvaginal digital examination in determining the position of foetal head in the birth canal. One of the studies found that the occiput posterior position was identified in $10 \%$, whereas in fact it was only present in $3 \%$ of cases. In $12 \%$ of cases, the midwives participating in the study were unable to determine the position of foetal head at all ${ }^{(12)}$. Other authors show that errors in the assessment of foetal head position are three times more common with the foetus in the occiput posterior position ${ }^{(10)}$. The findings presented above - combined with the fact that the delivery of foetus in the occiput posterior position is usually longer, and more frequently represents an indication to perform an obstetric intervention - provide additional evidence that transvaginal digital examination is characterised by low reliability and limited utility.

The problem grows in importance especially when over the course of labour doubts arise as to the dynamics of its progress. An accurate and quick decision on how to end the labour, made at the appropriate stage, is crucial both to maternal and foetal health. Rapid advancements in medicine have confronted obstetricians with a growing need to objectify the diagnostic tools used during labour and delivery. One attempt to address this need is the proposed application of intrapartum sonography.

\section{Application of intrapartum ultrasound}

Intrapartum ultrasound (labour ultrasound) is a type of ultrasound assessment performed during labour which, by measuring a set of parameters, aims to evaluate spatial relationships between the head of the foetus and the birth canal - including foetal head position, attitude, and station. Intrapartum sonography is performed using a transabdominal approach or, alternatively, ultrasound images are obtained by placing the probe between the patient's labia. The latter type of examination is referred to as translabial ultrasound or transperineal ultrasound (TPU). In both examination types, images are obtained in the sagittal or transverse planes ${ }^{(13)}$.

Intrapartum sonography does not require an advanced ultrasound system. Preferably, the ultrasound unit should be a compact, portable device equipped with a rechargeable battery for quick start-up and temporary operation without connection to an external power supply. The examination is performed using a low-frequency $(<4 \mathrm{MHz})$ convex-type probe generating images in 2D presentation. Intrapartum ultrasound is a typical bedside examination (Fig. 1).

Recent years have brought considerable interest in intrapartum sonography, resulting in a number of studies evaluating the application of the method to better assess the progress of labour and make correct clinical decisions. The available study data were compiled in the form of guidelines drawn up by the International Society of Ultrasound in Obstetrics and Gynecology (ISUOG), an international association of sonography experts ${ }^{(13)}$. The Practice Guidelines outline the clinical situations which may represent indications for performing ultrasound evaluation in labour. They include slow progress or arrest of labour, suspected foetal head malpresentation, or the need to ascertain foetal head position and station before considering or performing instrumental vaginal delivery. Among multiple parameters, the Practice Guidelines highlight the special role of the angle of progression (AoP), head-perineum distance (HPD), head direction (HD) and midline angle (MLA).

The angle of progression (AoP), also referred to as the angle of descent, is the angle between the long axis of the 


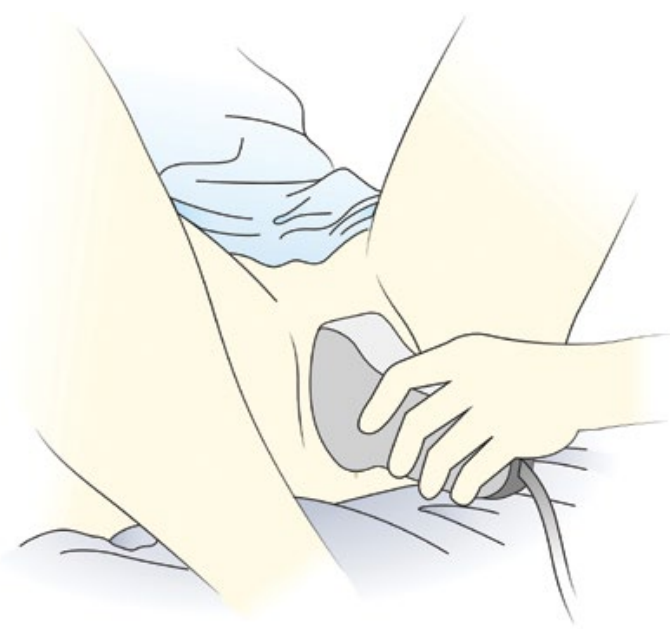

Fig. 1. Transperineal ultrasound

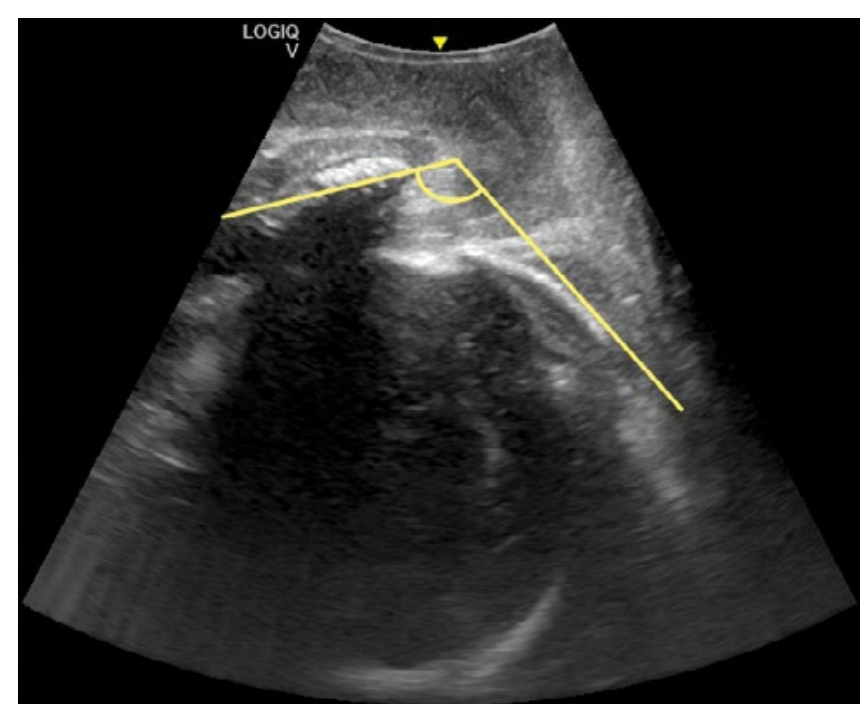

Fig. 2. TPU scan with marked angle of progression (AoP)

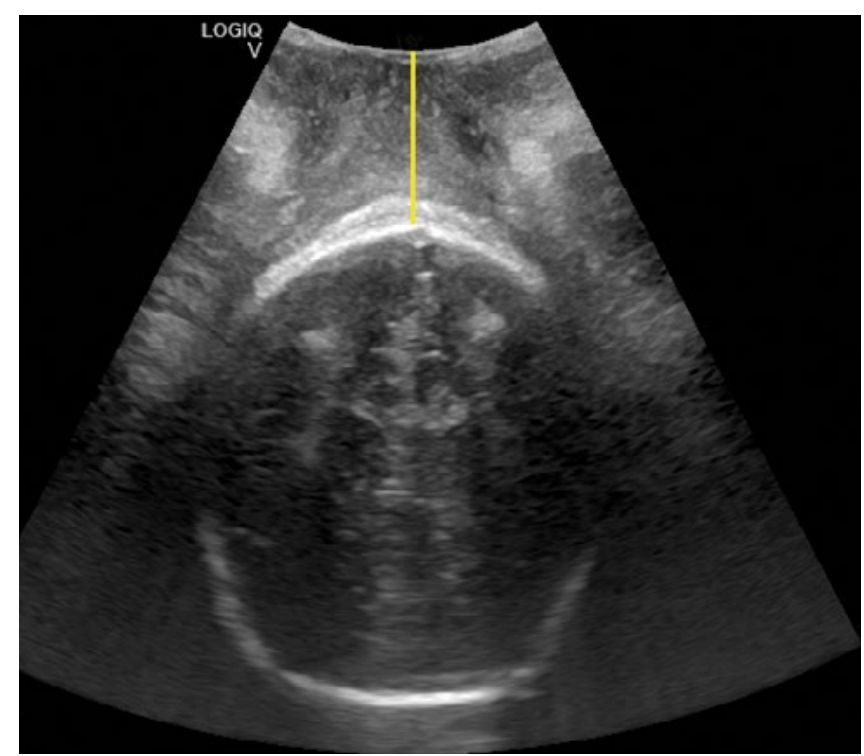

Fig. 3. TPU scan with yellow line marking the distance between foetal skull and maternal perineum (HPD) symphysis pubis and the line extending tangentially from its most inferior edge to the foetal skull(14) (Fig. 2). It is, as yet, the most thoroughly investigated parameter of TPU, characterised by high accuracy and repeatability ${ }^{(15-17)}$. It has also been found that foetal head station at the level of the interischial line corresponds to an AoP of $116^{\circ(18)}$, which is extremely useful information for the clinician managing the delivery.

Similarly to the AoP, the head-perineum distance (HPD) is a parameter determined from the transperineal approach. However, unlike the AoP, it is measured in the frontal (rather than sagittal) projection. The HPD is defined as the shortest distance from the outer bony limit of the foetal skull to the maternal perineum ${ }^{(19)}$ (Fig. 3).

It has been shown that based on the above parameters objective conclusions can be drawn about foetal head station in the birth canal, which is of particular significance for predicting the progress of labour and determining the patient's mode of delivery - instrumental or Caesarean section - if arrest of labour is diagnosed. A number of authors agree that the AoP $>120^{\circ}$ is associated with the highest probability of spontaneous vaginal delivery ${ }^{(14,18,20,21)}$. For the HPD, the correlation has been confirmed for values less than $40 \mathrm{~mm}^{(22)}$.

Midline angle (MLA) is a parameter evaluated, similarly to the HPD, in the frontal projection. It is defined as the angle between the anteroposterior axis of the maternal pelvis and the midline of the foetal brain visible as a hyperechogenic line interposed between the two cerebral hemispheres ${ }^{(23)}$ (Fig. 4). A change in MLA value reflects the turns the foetal head makes when negotiating through successive sections of the birth canal. In combination with the parameters defining the foetal head station, it accurately reflects the position of the head in the birth canal, so that if instrumental delivery is considered necessary, the obstetrician has access to valuable tips on how to effectively apply tractions to repair the abnormal birth mechanism.

The head direction (HD) is the angle between the longest recognisable axis of the foetal head and the long axis of the pubic symphysis (Fig. 5). Depending on the values of the angle, different categories of the parameter are distinguished:

- head up - when the angle is equal to or greater than $30^{\circ}$,

- horizontal - when the value of the angle is between 0 and $29^{\circ}$,

- head down - when the angle is less than $0^{\circ}$.

The change in the direction of foetal head as it descends in the birth canal reflects the orientation of the lead point along the curved axis of the birth canal - from turning "downward" through the horizontal direction to turning "upward"(24).

Similarly to the MLA, the HD result makes it possible to determine with a high degree of accuracy the safety, difficulty and successful outcome of instrumental vaginal delivery ${ }^{(18,24)}$. Foetal head direction "head up" combined with the 


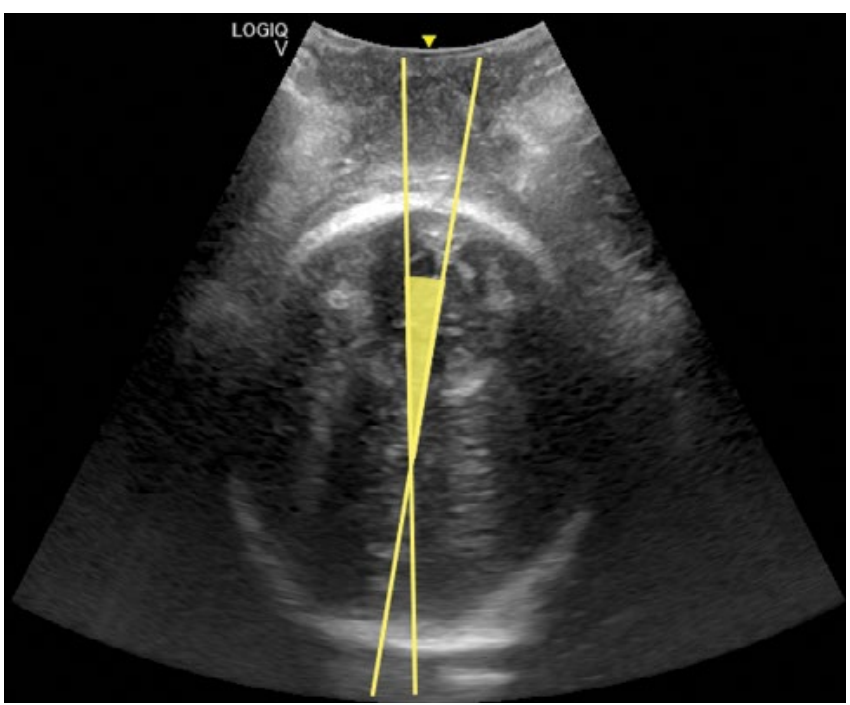

Fig. 4. TPU scan with marked midline angle (MLA)

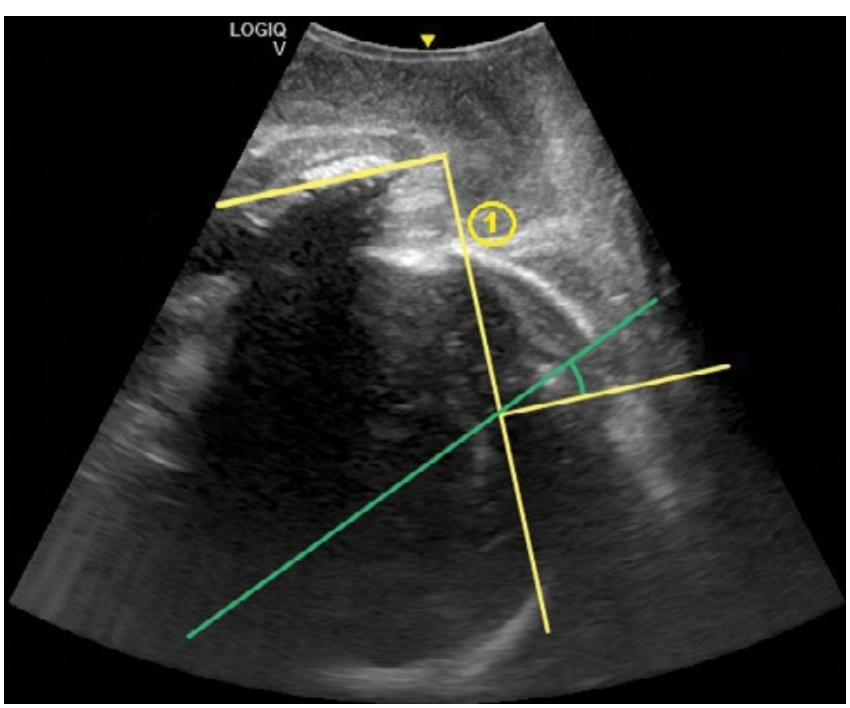

Fig. 5. TPU scan with visible subpubic line (1) and angle determining head direction (HD)

MLA of less than $45^{\circ}$ represent a good prognostic factor for the successful use of vacuum extraction or forceps ${ }^{(25)}$.

Over the past year, a number of promising study findings have been reported on the application of intrapartum sonography, providing a set of reliable data justifying widespread use of the method in the delivery room.

\section{Problems in the second stage of labour}

One of the situations which may raise considerable diagnostic doubts even in experienced obstetricians is unduly prolonged second stage of labour, when the key issue becomes the assessment of chance of natural delivery, so that a risky medical intervention can be avoided. Dall'Asta et al. ${ }^{(26)}$ evaluated the benefit of a range of TPU parameters including the AoP, MLA, HPD, and the head-symphysis distance (HSD, not discussed above), in the prediction of the mode of delivery among women diagnosed with failure to progress in the second stage of labour. The authors found that the only independent predictors of spontaneous vaginal delivery were the MLA and HSD (ROC of $80 \%$ and 74\%, respectively). These findings shed new light on the benefits of the HSD, whose diagnostic benefit has not, as yet, been demonstrated scientifically with a sufficient degree of reliability.

Although the ISUOG Practice Guidelines indicate how the findings obtained by intrapartum ultrasound can be useful in the objective qualification of patients for instrumental delivery, much research is still focused on attempts to identify precise cut-off points for different parameters in order to support clinicians in the decision-making process. This is because an erroneous choice of the above procedures in the second stage of labour is associated with potentially the highest number of complications, both maternal and foetal. In one large analysis, comprising a total of 116 instrumental deliveries, it was shown that the AoP value of at least $138.7^{\circ}$ and $160.9^{\circ}$ (measured between and during uterine contractions, respectively) was associated in a significant manner with the highest rate of successful instrumental deliveries ${ }^{(27)}$. Based on various sources, the AoP assessment is a good predictor (80-87\% probability) of a successful attempt to perform instrumental delivery ${ }^{(27,28)}$. Another study, which assessed the HPD, found that the duration of vacuum extraction was the shortest in the group of patients with the greatest difference between the HPD values measured during uterine contraction and relaxation ${ }^{(29)}$.

\section{Applications of intrapartum ultrasound in the perinatal period}

In the recent period, intrapartum ultrasound has been expanding its scope to include the perinatal period. This is because of a number of new publications addressing the issue of prediction of the mode of delivery even before the spontaneous or induced start of labour. Such prediction would significantly improve the quality of perinatal care through the early identification of patients who do not have a good chance of natural (vaginal) delivery. A prospective study evaluating the TPU parameters before the induction of labour in pregnant women at term showed that in addition to variables including maternal age or history of natural vaginal delivery, only the AoP was a significant independent prognostic factor for labour induction ${ }^{(30)}$. Another study, involving a total of 250 patients, assessed the HPD under similar conditions. The authors observed that a cut-off of $\leq 5.5 \mathrm{~cm}$ for the foetal head-perineum distance was associated with the highest predictability (sensitivity 97\%, specificity $88 \%$ ) of the successful outcome of induction ${ }^{(31)}$. Serial measurements of the TPU parameters - particularly the AoP - performed during labour induction can also be used as an objective method for recognising ineffective induction and lack of labour progress earlier than 
indicated by traditional transvaginal digital examination $^{(32)}$. Despite these optimistic results, the AoP has been shown to be of little value in predicting the delivery mode in patients undergoing induction of labour. At present, there is no sufficient evidence to show that incorporating measurements of this parameter into routine assessment determining the ending of pregnancy would help to identify patients in whom labour induction proves ineffective ${ }^{(30,33)}$.

Also, there have been interesting attempts to use the TPU parameters in the evaluation of indications for the hospitalisation of patients presenting with contractile activity. One study, performed on a small group of patients (57), showed that in the vast majority of cases (96.5\%), a decision to admit pregnant women at term to hospital due to labour was possible based solely on the TPU findings which were consistent with the results of manual gynaecological examination. The authors ventured the conclusion that TPU performed before admitting a patient makes it possible to avoid up to $90 \%$ of transvaginal digital examinations ${ }^{(34)}$. The method could be particularly useful in cases of false labour, when the patient frequently undergoes a number of unnecessary transvaginal digital examinations.

\section{Intrapartum ultrasound and the comfort of women in labour}

Other benefits of intrapartum sonography are also increasingly brought into focus. For example, TPU performed during maternal pushing has a positive impact on the effectiveness of contractions, and can be used as a visual biofeedback contributing to a shorter duration of labour ${ }^{(33)}$. Another important aspect that needs to be taken into account relates to the discomfort and pain experienced by patients who are made to undergo a series of transvaginal digital examinations in labour. In a study where the progress of labour was assessed both traditionally (per vaginam) and by means of intrapartum sonography, a survey of patients' opinions and experiences revealed that $87 \%$ of them would prefer to be examined solely by transperineal ultrasound during their next birth ${ }^{(35)}$. Obstetricians have an equally favourable opinion of the method. A survey of physicians participating in a course on the applications of intrapartum ultrasound showed that after completing the course more than twice as many participants as before the training were convinced of the usefulness of the method for evaluating foetal head station in the birth canal. In addition, the number of participants claiming that TPU is a complex examination decreased significantly ${ }^{(36)}$.

\section{Conclusions}

The arguments in favour of incorporating intrapartum ultrasound into regular obstetric practice are manifold. Initial studies have shown that intrapartum sonography is a more accurate method for the assessment of foetal head position than routine transvaginal digital examination.

Expanding the scope of gynaecological examination to include TPU findings may aid in the selection of mode of delivery, support the clinician in making safe decisions, and provide guidance on the need to perform operative delivery in the presence of the most experienced obstetrician, with the operating room ready for use in case of an emergency.

A number of arguments speak for integrating intrapartum ultrasound into routine clinical practice. Firstly, the examination is easy to perform ${ }^{(36)}$, and yields repeatable results ${ }^{(37)}$. It does not require advanced medical equipment and, as long as a portable ultrasound system is available, intrapartum sonography is an easily accessible, rapid bedside examination producing results in less than a few minutes. The time necessary for learning the method and achieving precision in its application is considerably shorter than in the case of gynaecological examination ${ }^{(38)}$, and the reliability of results does not depend on the level of experience in performing ultrasound examinations ${ }^{(17)}$. Intrapartum sonography is a painless examination ${ }^{(39,40)}$ and, in the opinion of patients, it is a highly acceptable form of monitoring the progress of labour, which enhances pregnant women's trust towards medical professionals ${ }^{(41)}$. Secondly, intrapartum ultrasound is useful from the viewpoint of medical and legal issues involved in obstetric interventions. Supplementing medical records with objective intrapartum ultrasound findings in the form of scan may help prove the validity of actions taken during labour ${ }^{(42)}$. It is hoped, though there is no evidence yet, that the use of intrapartum ultrasound will reduce the frequency of performing transvaginal digital examinations $^{(34)}$, and hence contribute to lowering the risk of intrauterine infections.

However, there are still many obstacles hindering the incorporation of results obtained by intrapartum ultrasound into routine labour management algorithms. Even though numerous studies have shown the method to be very precise, it is still not clear which of the parameters has the greatest clinical significance, or whether a more accurate approach would be to use the results obtained for several parameters - and if so, which ones. Furthermore, there are no recommendations as to when precisely intrapartum ultrasound should be performed during labour to yield the greatest diagnostic benefit. Attempts are also ongoing to identify the values of cutoff points for different parameters to assist obstetricians in making reliable clinical decisions. Answering these questions would require extensive randomized studies conducted in large groups of women in labour, which, given the complex nature of childbirth, might be difficult to achieve. Nevertheless, when learning this new method, it is worthwhile to look for correlations, comparing the results of own manual gynaecological examinations with TPU findings. Validation of the use of ultrasound techniques during labour, which was presented in 
the ISUOG Practice Guidelines ${ }^{(13)}$, creates a solid basis for collecting own observations and experiences related to the use of the method. In the future, such data may lead to the development of universally valid recommendations improving the quality of care provided to women in labour.

\section{References}

1. Hobbins JC: Overview of imaging in pregnancy: history to the present, including economic impact. Semin Perinatol 2013; 5: 290-291.

2. Goldberg BG: Obstetric US Imaging: The Past 40 Years, Radiology 2000; 215: 622-629.

3. Dębska M, Kretowicz P, Dębski R: Intrapartum sonography - eccentricity or necessity? J Ultrason 2015; 15: 125-136.

4. Phelps JY, Higby H, Smyth MH, Ward JA, Arredondo F, Mayer AR: Accuracy and intraobserver variability of simulated cervical dilatation measurements. Am J Obstet Gynecol 1995; 3: 942-945.

5. Huhn KA, Brost BC: Accuracy of simulated cervical dilation and effacement measurements among practitioners. Am J Obstet Gynecol 2004; 5: 1797-1799.

6. Buchmann EJ, Libhaber E: Accuracy of cervical assessment in the active phase of labour. BJOG 2007; 114: 833-837.

7. Dupuis O, Silveira R, Zentner A, Dittmar A, Gaucherand P, Cucherat M et al.: Birth simulator: reliability of transvaginal assessment of fetal head station as defined by the American College of Obstetricians and Gynecologists classification. Am J Obstet Gynecol 2005; 3: 868-874.

8. Sherer DM, Miodovnik M, Bradley KS, Langer O: Intrapartum fetal head position II: comparison between transvaginal digital examination and transabdominal ultrasound assessment during the second stage of labor. Ultrasound Obstet Gynecol 2002; 19: 264-268.

9. Sherer DM, Miodovnik M, Bradley KS, Langer O: Intrapartum fetal head position I: comparison between transvaginal digital examination and transabdominal ultrasound assessment during the active stage of labor. Ultrasound Obstet Gynecol 2002; 19: 258-263.

10. Souka AP, Haritos T, Basayiannis K, Noikokyri N, Antsaklis A: Intrapartum ultrasound for the examination of the fetal head position in normal and obstructed labor. J Matern Fetal Neonatal Med 2003; 13: 59-63.

11. Shetty J, Aahir V, Pandey D, Adiga P, Kamath A: Fetal head position during the first stage of labor: comparison between vaginal examination and transabdominal ultrasound. ISRN Obstet Gynecol 2014, 314617.

12. Dimassi K, Ben Amor A, Belghith C, Ben Khedija MA, Triki A, Gara MF: Ultrasound diagnosis of fetal head engagement. Int J Gynaecol Obstet 2014; 127: 6-9.

13. Ghi T, Eggebø T, Lees C, Kalache K, Rozenberg P, Youssef A et al:: ISUOG Practice Guidelines: intrapartum ultrasound. Ultrasound Obstet Gynecol 2018; 52: 128-139.

14. Barbera AF, Pombar X, Perugino G, Lezotte DC, Hobbins JC: A new method to assess fetal head descent in labor with transperineal ultrasound. Ultrasound Obstet Gynecol 2009; 33: 313-319.

15. Eggebø TM, Heien C, Økland I, Gjessing LK, Romundstad P, Salvesen KA: Ultrasound assessment of fetal head-perineum distance before induction of labor. Ultrasound Obstet Gynecol 2008; 32: 199-204.

16. Molina FS, Terra R, Carrillo MP, Puertas A, Nicolaides KH: What is the most reliable ultrasound parameter for assessment of fetal head descent? Ultrasound Obstet Gynecol 2010; 36: 493-499.

17. Dückelmann AM, Bamberg C, Michaelis SA, Lange J, Nonnenmacher A, Dudenhausen JW et al:: Measurement of fetal head descent using the 'angle of progression' on transperineal ultrasound imaging is reliable regardless of fetal head station or ultrasound expertise. Ultrasound $\mathrm{Ob}$ stet Gynecol 2010; 35: 216-222.

18. Tutschek B, Braun T, Chantraine F, Henrich W: A study of progress of labour using intrapartum translabial ultrasound, assessing head station, direction, and angle of descent. BJOG 2011; 118: 62-69.

\section{Conflict of interest}

Authors do not report any financial or personal connections with other persons or organizations, which might negatively affect the contents of this publication and/or claim authorship rights to this publication.
19. Eggebø TM, Gjessing LK, Heien C, Smedvig E, Økland I, Romundstad P et al.: Prediction of labor and delivery by transperineal ultrasound in pregnancies with prelabor rupture of membranes at term. Ultrasound Obstet Gynecol 2006; 27: 387-391.

20. Kalache KD, Dückelmann AM, Michaelis SA, Lange J, Cichon G, Dudenhausen JW: Transperineal ultrasound imaging in prolonged second stage of labor with occipitoanterior presenting fetuses: how well does the 'angle of progression' predict the mode of delivery? Ultrasound Obstet Gynecol 2009; 33: 326-330.

21. Nishimura K, Yoshimura K, Kubo T, Hachisuga T: Objective diagnosis of arrested labor on transperineal ultrasound. J Obstet Gynaecol Res 2016; 42: 803-809.

22. Eggebø TM, Hassan WA, Salvesen KA, Lindtjørn E, Lees CC: Sonographic prediction of vaginal delivery in prolonged labor: a two-center study. Ultrasound Obstet Gynecol 2014; 43: 195-201.

23. Ghi T, Farina A, Pedrazzi A, Rizzo N, Pelusi G, Pilu G: Diagnosis of station and rotation of the fetal head in the second stage of labor with intrapartum translabial ultrasound. Ultrasound Obstet Gynecol 2009; 33: 331-336.

24. Henrich W, Dudenhausen J, Fuchs I, Kämena A, Tutschek B: Intrapartum translabial ultrasound (ITU): sonographic landmarks and correlation with successful vacuum extraction. Ultrasound Obstet Gynecol 2006; 28: 753-760.

25. Sainz JA, Borrero C, Aquise A, Serrano R, Gutiérrez L, FernándezPalacín A: Utility of intrapartum transperineal ultrasound to predict cases of failure in vacuum extraction attempt and need of cesarean section to complete delivery. J Matern Fetal Neonatal Med 2016; 29: 1348-1352.

26. Dall'Asta A, Angeli L, Masturzo B, Volpe N, Luca Schera GB, Di Pasquo E et al:: Prediction of spontaneous vaginal delivery in nulliparous women with a prolonged second stage of labor: the value of intrapartum ultrasound. Am J Obstet Gynecol 2019; pii: S0002-9378(19)31211-6.

27. Chan VYT, Lau WL, So MKP, Leung WC: Measuring angle of progression by transperineal ultrasonography to predict successful instrumental and cesarean deliveries during prolonged second stage of labor. Int J Gynaecol Obstet 2019; 144: 192-198.

28. Sainz JA, García-Mejido JA, Aquise A, Borrero C, Bonomi MJ, Fernández-Palacín A: A simple model to predict the complicated operative vaginal deliveries using vacuum or forceps. Am J Obstet Gynecol 2019; 220: 193.e1-193.e12.

29. Kahrs BH, Usman S, Ghi T, Youssef A, Torkildsen EA, Lindtjørn E et al.: Descent of fetal head during active pushing: secondary analysis of prospective cohort study investigating ultrasound examination before operative vaginal delivery. Ultrasound Obstet Gynecol 2019; 54: 524-529.

30. Chan WWY, Chaemsaithong P, Lim WT, Tse AWT, Kwan AHW, Leung TY et al:: Pre-induction transperineal ultrasound assessment for the prediction of labor outcome. Fetal Diagn Ther 2019; 45: 256-267.

31. Ali J, Hebbar S: Ultrasound assessment of foetal head-perineum distance prior to induction of labour as a predictor of successful vaginal delivery. J Obstet Gynaecol India 2019; 69: 129-135.

32. Tse WT, Chaemsaithong P, Chan WWY, Kwan AHW, Huang J, Appiah K et al.: Labor progress determined by ultrasound is different in women requiring cesarean delivery from those who experience a vaginal delivery following induction of labor. Am J Obstet Gynecol 2019; 221: 335. e1-335.e18.

33. Youssef A, Dodaro MG, Montaguti E, Consolini S, Ciarlariello S, Farina A et al.: Dynamic changes of fetal head descent at term before the 
onset of labor correlate with labor outcome and can be improved by ultrasound visual feedback. J Matern Fetal Neonatal Med 2019; 8: 1-8.

34. Cuerva MJ, García-Casarrubios P, García-Calvo L, Gutiérrez-Simon M, Ordás P, Magdaleno F et al.: Use of intrapartum ultrasound in term pregnant women with contractions before hospital admission. Acta Obstet Gynecol Scand 2019; 98: 162-166.

35. Mohan A, Mittal P, Bharti R, Grover SB, Suri J, Mohan U: Assessment of labor progression by intrapartum ultrasonography among term nulliparous women. Int J Gynaecol Obstet 2019; 147: 78-82.

36. Youssef A, Kamel R: Ultrasound in labor: impact of a theoretical and practical course on caregiver's perspective and accuracy. J Matern Fetal Neonatal Med 2019; 30: 1-7.

37. Sainz JA, Fernández-Palacín A, Borrero C, Aquise A, Ramos Z, GarcíaMejido JA: Intra and interobserver variability of intrapartum transperineal ultrasound measurements with contraction and pushing. J Obstet Gynaecol 2018; 38: 333-338.
38. Rozenberg P, Porcher R, Salomon LJ, Boirot F, Morin C, Ville Y: Comparison of the learning curves of digital examination and transabdominal sonography for the determination of fetal head position during labor. Ultrasound Obstet Gynecol 2008; 31: 332-337.

39. Chan YT, Ng KS, Yung WK, Lo TK, Lau WL, Leung WC: Is intrapartum translabial ultrasound examination painless? J Matern Fetal Neonatal Med 2016; 29: 3276-3280.

40. Usman S, Barton H, Wilhelm-Benartzi C, Lees CC: Ultrasound is better tolerated than vaginal examination in and before labour. Aust $\mathrm{N} \mathrm{Z} \mathrm{J}$ Obstet Gynaecol 2019; 59: 362-366.

41. Iliescu DG, Tudorache S, Cara ML, Dragusin R, Carbunaru O, Florea M et al.: Acceptability of intrapartum ultrasound monitoring - experience from a Romanian Longitudinal Study. Curr Health Sci J 2015; 41: 355-360.

42. Malvasi A, Montanari Vergallo G, Tinelli A, Marinelli E: „Can the intrapartum ultrasonography reduce the legal liability in distocic labor and delivery?" J Matern Fetal Neonatal Med 2018; 31: 1108-1109. 